\title{
Estruturas de variância residual para estimação de funções de covariância para o peso de bovinos da raça Canchim ${ }^{1}$
}

\author{
Fábio Luiz Buranelo Toral ${ }^{2}$, Maurício Mello de Alencar ${ }^{3}$, Alfredo Ribeiro de Freitas ${ }^{3}$ \\ 1 Projeto financiado pela EMBRAPA e FAPESP. \\ 2 Universidade Federal de Mato Grosso. \\ ${ }^{3}$ Embrapa Pecuária Sudeste. Bolsista do CNPq.
}

RESUMO - Este trabalho foi realizado com o objetivo de avaliar a utilização de diferentes estruturas de variância residual para estimação de funções de covariância para o peso de bovinos da raça Canchim. As funções de covariância foram estimadas pelo método da Máxima Verossimilhança Restrita em um modelo animal com os efeitos fixos de grupo de contemporâneos (ano e mês de nascimento e sexo), idade da vaca ao parto como covariável (efeitos linear e quadrático) e da trajetória média de crescimento, enquanto os efeitos aleatórios considerados foram os efeitos genéticos aditivos direto e materno, de ambiente permanente individual e materno e residual. Foram utilizadas diversas estruturas para a variância residual: funções de variâncias de ordem linear até quíntica e 1, 5, 10, 15 ou 20 classes de idades. A utilização de variância residual homogênea não foi adequada. A utilização da função de variância residual quártica e a divisão da variância residual em 20 classes proporcionaram os melhores ajustes, e a divisão em classes foi mais eficiente que a utilização de funções. As estimativas de herdabilidade direta se situaram entre 0,16 e 0,25 na maioria das idades consideradas e as maiores estimativas foram obtidas próximo aos 360 dias de idade e no final do período estudado. Em geral, as estimativas de herdabilidade direta foram semelhantes para os modelos com variância residual homogênea, função de variância residual quártica ou com 20 classes de idade. A melhor descrição das variâncias residuais para o peso em diversas idades de bovinos da raça Canchim foi a que considerou 20 classes heterogêneas. Entretanto, como existem classes com variâncias semelhantes, é possível agrupar algumas delas e reduzir o número de parâmetros estimados.

Palavras-chave: correlação, herdabilidade, regressão aleatória

\section{Residual variance structures to estimate covariance functions for weight of Canchim beef cattle}

\begin{abstract}
This study was carried out to evaluate the use of different residual variance structures to estimate covariance functions for weight of Canchim beef cattle. The covariance functions were estimated by the Restricted Maximum Likelihood method, in an animal model with fixed effect of contemporary group (year and month of birth and sex), age of dam at calving as covariable (linear and quadratic effects) and mean growth trajectory while the random effects considered were the direct additive genetic and maternal effects, permanent individual environment and maternal and residual effects. Different structures were used for residual variance: ordinary polynomials of different orders (linear to fifth) and step functions with 1, 5, 10, 15 and 20 classes. Homogeneous residual variance was not adequate. The four-ordered variance function and the step function with 20 classes presented the best fit and the class division was more efficient than residual functions. The estimated heritabilities were between 0.16 and 0.25 , and the greatest ones were obtained close to 360 days and at the end of the study period. The estimated heritabilities were similar for models with homogeneous residual variance, four-ordered residual function and step model with 20 classes of heterogeneous variances. The best description of residual variance, for weights at different ages of Canchim beef cattle, was by step function with 20 classes of heterogeneous variances. However, as existing classes with the same variances, they can be grouped to reduce the number of parameters to be estimated.
\end{abstract}

Key Words: correlation, heritability, random regression

\section{Introdução}

Os modelos de regressão aleatória (MRA) permitem a avaliação da trajetória de crescimento dos animais utilizando-se todos os dados disponíveis e modelando as variâncias ao longo do tempo. As acurácias para os valores genéticos preditos com MRA podem ser superiores àquelas obtidas com modelos de características múltiplas 
(Meyer, 2004). Nas análises de características múltiplas, as variâncias genéticas e residuais são obtidas para cada característica, enquanto nos MRA são utilizadas as funções de covariância (FC) para estimá-las.

Os aumentos da média e do desvio-padrão e as reduções do número de dados e da precisão das medidas de peso, que ocorrem com o avançar da idade, podem proporcionar alterações nas variâncias, especialmente na variância residual. Modelar adequadamente a variância residual ao longo da trajetória de crescimento é importante para que as estimativas dos demais parâmetros sejam adequadas. O agrupamento das idades em classes com variâncias residuais semelhantes (El Faro \& Albuquerque, 2003) e a utilização de funções de variância (Albuquerque \& Meyer, 2001; El Faro \& Albuquerque, 2003) têm sido utilizados para modelar variâncias residuais.

O número de parâmetros estimados para a avaliação genética, considerando variâncias residuais heterogêneas, pode ser elevado e constitui um entrave para a adoção de MRA. Modelos parcimoniosos são obtidos sem o comprometimento do ajuste e das demais funções de covariância e podem contribuir para o aumento da utilização de MRA nas avaliações genéticas para peso. Neste contexto, este trabalho foi realizado com o objetivo de avaliar a utilização de diferentes estruturas de variância residual para a estimação de funções de covariâncias para o peso de bovinos da raça Canchim.

\section{Material e Métodos}

Foram utilizados os dados de pesos de bovinos da raça Canchim pertencentes ao rebanho da Embrapa Pecuária Sudeste, localizada no município de São Carlos, São Paulo. Os animais nasceram entre 1954 e 1978, exceto 1955, e foram criados exclusivamente em pastagens com suplementação mineral. Neste período, os animais foram pesados mensalmente, entre os dois últimos e os dois primeiros dias úteis de cada mês. Maiores informações sobre as condições de alimentação e manejo dos animais foram descritas por Mello et al. (2002).

Apenas os dados de animais com 9 a 25 pares de medidas peso-idade, tomadas entre o $15^{\circ}$ e o $760^{\circ}$ dia de vida foram considerados. Os pesos ao nascimento não foram utilizados para evitar a existência de idades (1 a 14 dias) sem nenhum par de medidas peso-idade e porque, em análises preliminares utilizando esse peso, observaram-se correlações e covariâncias aparentemente incoerentes entre os pesos ao nascimento e às demais idades. Os dados de animais pertencentes a grupos de contemporâneos (ano e mês de nascimento e sexo) com menos de dez laços genéticos de ligação (Fries \& Roso, 1998) com outro grupo e com menos de três pais diferentes não foram considerados. Após essas restrições, formou-se um arquivo com os dados de 2.399 animais, dos quais $16 \%$ possuíam 15, ou menos, medidas de peso-idade, enquanto os demais possuíam 16, ou mais, medidas de peso-idade, totalizando 48.077 pares de medidas.

Os efeitos genéticos aditivos direto e materno, de ambiente permanente individual e materno e a trajetória média de crescimento da população foram modelados utilizando-se polinômios ortogonais de Legendre de ordem 5 (intercepto, linear, quadrático, cúbico e quártico). O modelo geral considerado foi o seguinte:

$y_{i j l v}=G C_{l}+b_{1}\left(i v_{v}-\overline{i v}\right)+b_{2}\left(i v_{v}-\overline{i v}\right)^{2}+\sum_{k=0}^{4} f_{k} \phi_{k}\left(i d_{i j}^{*}\right)+$

$\sum_{k=0}^{4} a_{i k} \phi_{k}\left(i d_{i j}^{*}\right)+\sum_{k=0}^{4} m_{v k} \phi_{k}\left(i d_{i j}^{*}\right)+\sum_{k=0}^{4} c_{i k} \phi_{k}\left(i d_{i j}^{*}\right)+\sum_{k=0}^{4} q_{v k} \phi_{k}\left(j d_{i j}^{*}\right)+e_{i j l v}$, em que: $y_{i j l v}=$ peso do animal $i$ na idade $j$ filho da vaca $v$ e criado no grupo de contemporâneos $l$; $G C_{l}=$ efeito do grupo de contemporâneos (ano e mês de nascimento e sexo) $l ; b_{1}$ e $b_{2}=$ coeficientes linear e quadrático da idade da vaca $v$ sobre o peso do bezerro; $i v_{v}=$ idade da vaca $v$ no dia do parto do animal $i ; \overline{i v}=$ média de idade das vacas ao parto; $i d_{i j}^{*}=$ idade $j$ padronizada ( -1 a 1 ) do animal $i ; f_{k}=$ coeficientes da regressão fixa utilizada para descrever a trajetória média de crescimento da população; $\phi_{k}\left(i d_{i j}^{*}\right)=$ polinômios de Legendre para as idades padronizadas; $a_{i k}, m_{v k}, c_{i k} \mathrm{e}$ $q_{v k}=$ coeficientes aleatórios genéticos aditivos direto do animal $i$ e materno da vaca $v$, de ambiente permanente individual do animal $i$ e materno da vaca $v$, respectivamente; e $e_{i j l v}=$ resíduo associado a cada observação. Sob a forma matricial, esse modelo pode ser representado por:

$y=X b+Z_{1} a+Z_{2} m+Z_{3} c+Z_{4} q+e$,

em que: $y=$ vetor com os 48.077 pesos corporais, medidos em 2.399 animais; $X=$ matriz de incidência dos efeitos fixos (48.077 × 188), sendo 181 grupos de contemporâneos, dois coeficientes para os efeitos linear e quadrático da idade da vaca e cinco coeficientes para o efeito da trajetória média de crescimento; $b=$ vetor das soluções para os efeitos fixos $(188 \times 1)$; $Z_{1}(48.077 \times 11.995), Z_{2}(48.077 \times$ 4.375), $Z_{3}(48.077 \times 11.995)$ e $Z_{4}(48.077 \times 4.375)=$ matrizes de incidência dos efeitos aleatórios genéticos aditivos direto e materno e de ambiente permanente individual e materno, respectivamente; $a(11.995 \times 1), m(4.375 \times 1), c$ $(11.995 \times 1)$ e $q(4.375 \times 1)=$ vetores das soluções dos efeitos aleatórios; e $e(48.077 \times 1)=$ vetor dos resíduos associados a cada observação. Foram assumidas as seguintes pressuposições sobre os efeitos aleatórios: 


$\left[\begin{array}{c}a \\ m \\ p \\ q \\ e\end{array}\right] \sim N M V\left\{\left[\begin{array}{l}0 \\ 0 \\ 0 \\ 0 \\ 0\end{array}\right],\left[\begin{array}{ccccc}K_{a} \otimes A & 0 & 0 & 0 & 0 \\ 0 & K_{m} \otimes A & 0 & 0 & 0 \\ 0 & 0 & K_{c} \otimes I_{2.399} & 0 & 0 \\ 0 & 0 & 0 & K_{q} \otimes I_{875} & 0 \\ 0 & 0 & 0 & 0 & R\end{array}\right]\right\}$,

em que: $K_{a}, K_{m}, K_{c}$ e $K_{q}=$ matrizes de covariâncias de ordem $5 \times 5$ entre os coeficientes aleatórios genéticos aditivos direto e materno e de ambiente permanente individual e materno; $A=$ matriz de parentesco $(4.251 \times 4.251) ; I_{2.399}$ e $I_{875}=$ matrizes identidade cujas ordens correspondem ao número de animais e de vacas com dados dos filhos; e $R$ = matriz de variâncias residuais.

Foram avaliadas diferentes estruturas para $R$, representadas como: a) $R=I_{746} \sigma_{e j}^{2}$, de modo que $\sigma_{e j}^{2}=\exp \left\{\sum_{r=0}^{o} b_{r}\left(i d_{j}^{*}\right)^{r}\right\}$, em que: $\sigma_{e j}^{2}=$ variância residual na idade $j ; b_{r}=$ coeficientes de ordem $r(o=1$ a 5 , intercepto + linear, ..., intercepto + linear até quíntico); $o$ = número de coeficientes das funções de variâncias residuais, além do intercepto; e $i d_{j}^{*}=$ idade $j$ padronizada para o intervalo entre -1 e 1 ; e b) $R=I_{746} \sigma_{e c}^{2}$, em que $\sigma_{e c}^{2}=$ variância residual para cada classe de variância $c(c=1,5,10,15$ e 20). Quando da utilização de classes de idades para modelar a variância residual, as classes foram formadas agrupando-se as idades da seguinte forma: uma classe (variâncias homogêneas), cinco classes (classe 1: 15 a 160 dias, classe 2: 161 a 310 dias, ..., classe 5: 611 a 760 dias), 10 classes (classe 1: 15 a 85 dias, classe 2: 86 a 160 dias, ..., classe 10: 686 a 760 dias), 15 classes (classe 1: 15 a 60 dias, classe 2: 61 a 110 dias, ..., classe 15: 711 a 760 dias) e 20 classes (classe $1: 15$ a 38, classe 2: 39 a $76, \ldots$, classe 20: 723 a 760 dias).

As funções de covariância foram estimadas por meio do método da Máxima Verossimilhança Restrita, e da opção DXMRR do programa DFREML (Meyer, 1998).

Para escolher a estrutura de variância residual mais adequada para a análise das funções de covariâncias, foram calculados os valores das funções de verossimilhança restrita (-2RLL) e os critérios de informação de Akaike (AIC), bayesiano de Schuwarz (BIC), e o de Akaike consistente (CAIC), de acordo com Bozdogan (1987) e Wolfinger (1993):

$$
\begin{aligned}
& -2 R L L=(n-p) \ln (2 \pi)+\ln |V|+\ln \left|X^{\prime} V^{-1} X\right|+(y-X b)^{\prime} \\
& V^{-1}(y-X b) ; \\
& A I C=-2 R L L+2 d ; \\
& B I C=-2 R L L+d \ln n ; \mathrm{e} \\
& C A I C=-2 R L L+d(\ln k+1),
\end{aligned}
$$

em que: $n$, representa o número de observações analisadas; $p$, o posto da matriz $X$; $V$, a matriz de covariância de $y$; $d$, o número de parâmetros do modelo; e os demais componentes das fórmulas foram definidos anteriormente. Valores de -2RLL, AIC, BIC e CAIC mais próximos de zero indicam melhor ajuste. Para comparação dos modelos, os critérios AIC, BIC e CAIC foram preferidos porque permitem a comparação de modelos não-aninhados e penalizam modelos com maior número de parâmetros (Wonfinger, 1993). O teste da razão de verossimilhança, além de ser indicado apenas para comparar modelos perfeitamente aninhados (Sorensen \& Gianola, 2002), tende a favorecer modelos com maior número de parâmetros (Meyer, 2000).

\section{Resultados e Discussão}

O polinômio de Legendre de ordem 5 (intercepto, linear, quadrático, cúbico e quártico) proporcionou bom ajuste aos dados. Entretanto, nas idades iniciais e finais, ocorreram pontos sub e superestimados, respectivamente (Figura 1). Em geral, polinômios de Legendre cúbicos (Albuquerque \& Meyer, 2001; Nobre et al., 2003; Dias et al., 2006), ou quárticos (Santoro et al., 2005) têm sido satisfatórios para modelar a trajetória de crescimento de zebuínos até 18 a 24 meses de idade.

O modelo com variâncias homogêneas (uma classe) proporcionou o pior ajuste entre os modelos considerados (Tabela 1). Este resultado está de acordo com o observado por Albuquerque \& Meyer (2001), Nobre et al. (2003), Sakaguti et al. (2003), Cyrillo (2003) e Dias et al. (2006), que também sugerem que as variâncias residuais não são constantes. Possíveis explicações para a alteração das variâncias residuais são o menor número e a maior dificuldade de obter as medidas em animais mais velhos. Além disso,

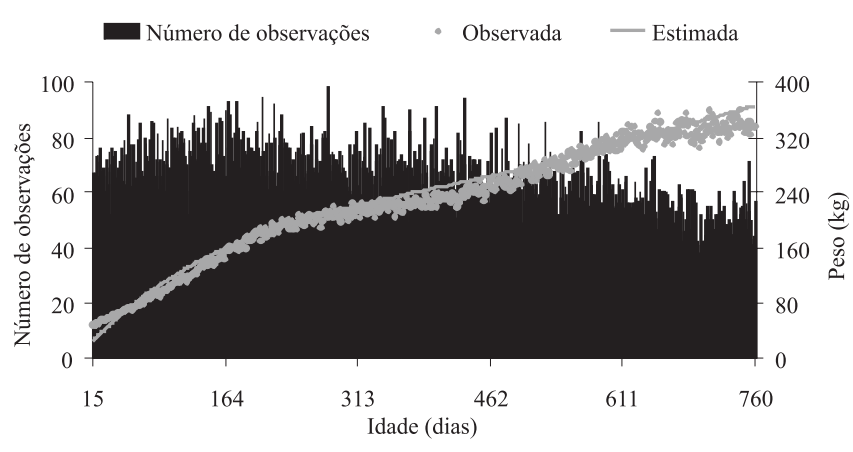

Figura 1 - Número de observações e médias observadas e estimadas dos pesos de bovinos da raça Canchim utilizando-se um polinômio de Legendre de ordem 5 para modelar a trajetória média de crescimento. 
Tabela 1 - Número de parâmetros, valor da função de máxima verossimilhança restrita e critérios de informação de Akaike, bayesiano de Schwarz e de Akaike consistente para cada modelo

\begin{tabular}{|c|c|c|c|c|c|}
\hline & \multirow{2}{*}{$\begin{array}{l}\text { Número de } \\
\text { parâmetros }\end{array}$} & \multirow{2}{*}{$\begin{array}{c}\text { Valor da função de máxima } \\
\text { verossimilhança restrita (-2RLL) }\end{array}$} & \multicolumn{3}{|c|}{ Critério de informação } \\
\hline & & & Akaike & Bayesiano de Schwarz & $\overline{\text { Akaike consistente }}$ \\
\hline \multicolumn{6}{|c|}{ Modelos com funções de variâncias residuais } \\
\hline Linear & 62 & 335,136 & 335,260 & 335,804 & 335,866 \\
\hline Quadrática & 63 & 335,136 & 335,262 & 335,815 & 335,878 \\
\hline Cúbica & 64 & 335,078 & 335,206 & 335,768 & 335,832 \\
\hline Quártica & 65 & 334,954 & 335,084 & 335,655 & 335,720 \\
\hline Quíntica & 66 & 334,954 & 335,086 & 335,666 & 335,732 \\
\hline \multicolumn{6}{|c|}{ Modelos com classes de variâncias residuais } \\
\hline 1 & 61 & 337,323 & 337,445 & 337,981 & 338,042 \\
\hline 5 & 65 & 335,104 & 335,234 & 335,805 & 335,870 \\
\hline 10 & 70 & 334,307 & 334,447 & 335,062 & 335,132 \\
\hline 15 & 75 & 334,047 & 334,197 & 334,856 & 334,931 \\
\hline 20 & 80 & 332,611 & 332,771 & 333,473 & 333,553 \\
\hline
\end{tabular}

alguns animais podem receber tratamentos diferenciados ao longo da vida sem que esses tratamentos sejam corretamente considerados.

Os valores dos critérios de ajuste obtidos com a utilização da função de variância residual quíntica foram maiores que aqueles obtidos com a função quártica, exceto para o valor da função de máxima verossimilhança restrita (-2RLL), no qual as duas foram equivalentes (Tabela 1). Quando utilizadas funções para modelar a variância residual, não foi necessária a utilização de funções com coeficientes superiores ao quártico. Albuquerque \& Meyer (2001), estudando os dados de pesos de bovinos da raça Nelore do nascimento aos 630 dias de idade com a utilização de modelos de regressão aleatória, modelaram os resíduos por meio de funções de variâncias cúbicas (intercepto, linear, quadrático e cúbico) e encontraram bons resultados, sugerindo que a utilização de funções de variâncias residual pode ser aplicada com a vantagem de exigir a estimação de menor número de parâmetros. Dias et al. (2006), trabalhando com dados de peso de bovinos Tabapuã do nascimento aos 550 dias de idade, também encontraram diferenças pequenas nos valores de AIC e BIC quando utilizaram modelos de regressão aleatória com funções de variância residual cúbica ou quártica, mas verificaram que a utilização da função de variância quíntica proporcionou melhor ajuste em relação aos modelos de menor ordem. Estes autores verificaram que modelos com ordens superiores a quíntica não proporcionaram melhoria significativa no ajuste.

Nas análises com a utilização de classes de variâncias residuais, os modelos com maior número de classes foram mais adequados (Tabela 1) e os resultados sugerem a necessidade de se trabalhar com modelos de mais de 20 classes para se determinar o número ótimo de classes utilizando funções de covariâncias, como realizado por
El Faro \& Albuquerque (2003) ao estudarem a variância residual para a análise de dados do controle semanal de produção de leite em vacas da raça Caracu, com a variância residual dividida em até 43 classes, uma para cada semana de controle. Todavia, Cyrillo (2003) utilizou no máximo oito classes de variâncias residuais para modelar o efeito de ambiente temporário para o peso de bovinos Nelore com até 13 meses de idade e obtiveram resultados satisfatórios para os demais componentes de covariância, sugerindo que um número muito superior de classes, em relação ao utilizado neste estudo, não seria necessário.

Com a utilização da função de variância quártica ou 20 classes de idade para modelar a variância residual, houve tendência de aumento da variância com o aumento da idade (Figura 2). Contudo, esse comportamento foi irregular quando estimadas variâncias residuais específicas para cada uma das 20 classes de idade, especialmente na primeira classe de idades (15 a 38 dias de idade). O polinômio de Legendre utilizado para modelar a trajetória média de crescimento subestimou os pesos na primeira classe de

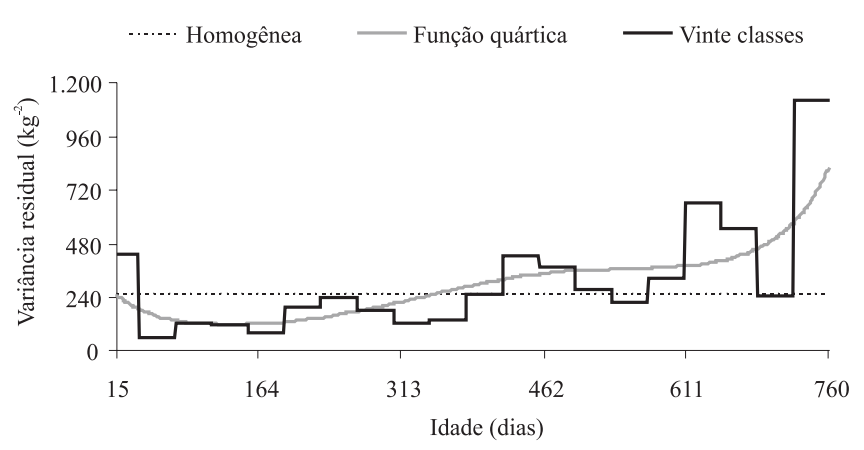

Figura 2 - Variâncias residuais para os pesos de bovinos da raça Canchim, de acordo com a estrutura de variância residual. 
idades em 16 kg (ou 30\%), em média (Figura 1), e esse fato contribuiu para obtenção de uma estimativa de variância residual maior que a esperada para esta classe.

Cyrillo (2003) observou aumento gradual da variância residual com a utilização de oito classes de idade à medida que a idade aumentou. Essa tendência também foi observada neste trabalho quando utilizadas apenas dez classes de idade para modelar a variância residual. Contudo, o modelo com maior número de classes de idade se ajustou melhor aos dados. Melhores estimativas da variância residual para cada classe são esperadas à medida que as amplitudes de idades nas classes são reduzidas, desde que as reduções dos números de observações nas classes não sejam proporcionais.

O agrupamento de classes (Figura 2) poderia ser uma alternativa para reduzir o número de parâmetros do modelo. Classes de idades adjacentes e com variâncias semelhantes (por exemplo: classes 3 e 4; 6, 7 e 8; e 9 e 10) poderiam ser reunidas em classes com maior amplitude de idades, desde que isso não comprometesse o ajuste do modelo.

A comparação dos critérios valor da função de máxima verossimilhança restrita e critérios de informação de Akaike, bayesiano de Schwarz e Akaike consistente proporcionados pelo modelo com função de variância residual quártica com aqueles proporcionados pelo modelo com 20 classes de variâncias residuais mostrou que este último modelo foi mais adequado que o primeiro para modelar a variância residual (Tabela 1), todavia exige estimação de maior número de parâmetros. Este resultado confirma relatos de El Faro \& Albuquerque (2003) de que os modelos com classes de variâncias são superiores aos modelos com funções de variâncias para modelagem das variâncias residuais no estudo da curva de lactação de vacas da raça Caracu. Conforme sugerido por Meyer (2000), o melhor ajuste do modelo com 20 classes de variâncias residuais, em relação aos modelos concorrentes, deve ser consequência de sua maior parametrização.

O comportamento da variância genética aditiva direta e da variância do efeito de ambiente permanente individual foi crescente ao longo das idades e semelhante entre as três estruturas de variâncias residuais (Figura 3), o que era esperado, uma vez que as variâncias e covariâncias entre os coeficientes das regressões aleatórias para estes efeitos também foram semelhantes (Tabela 2). Conforme relatado por Meyer (2000), as diferenças nas estruturas da variância residual têm maiores reflexos na variância fenotípica que em suas componentes.

Tabela 2 - Estimativas de covariâncias (diagonal e acima) e correlações (abaixo da diagonal) entre os coeficientes de regressão aleatória para os efeitos genéticos aditivos direto e materno e de ambiente permanente individual e materno com diferentes estruturas para a variância residual

\begin{tabular}{|c|c|c|c|c|c|c|c|c|c|c|}
\hline \multirow[t]{2}{*}{ Coeficiente $^{1}$} & \multicolumn{5}{|c|}{ Função de variância residual quártica } & \multicolumn{5}{|c|}{ Vinte classes de variâncias residuais } \\
\hline & I & $\mathrm{L}$ & Q2 & $\mathrm{C}$ & Q4 & I & $\mathrm{L}$ & Q2 & C & Q4 \\
\hline & \multicolumn{10}{|c|}{ Efeito genético aditivo direto } \\
\hline I & 596,687 & 225,233 & $-67,858$ & 58,819 & 55,444 & 577,632 & 218,170 & $-58,554$ & 64,190 & 62,521 \\
\hline $\mathrm{L}$ & 0,713 & 167,208 & 24,121 & 13,062 & 30,426 & 0,690 & 173,273 & 29,447 & 19,375 & 39,486 \\
\hline Q2 & $-0,389$ & 0,261 & 50,927 & $-1,256$ & $-5,999$ & $-0,345$ & 0,317 & 49,725 & 1,446 & $-0,088$ \\
\hline $\mathrm{C}$ & 0,433 & 0,182 & $-0,032$ & 30,872 & 18,774 & 0,426 & 0,235 & 0,033 & 39,366 & 28,210 \\
\hline \multirow[t]{2}{*}{ Q4 } & 0,361 & 0,374 & $-0,134$ & 0,538 & 39,490 & 0,360 & 0,415 & $-0,002$ & 0,621 & 52,354 \\
\hline & \multicolumn{10}{|c|}{ Efeito genético aditivo materno } \\
\hline I & 342,878 & 113,022 & $-27,224$ & 29,607 & 7,613 & 311,330 & 110,503 & $-22,399$ & 31,083 & 13,202 \\
\hline $\mathrm{L}$ & 0,927 & 43,313 & $-8,318$ & 8,956 & 5,951 & 0,944 & 43,995 & $-7,312$ & 9,635 & 7,750 \\
\hline Q2 & $-0,953$ & $-0,819$ & 2,382 & $-2,231$ & $-0,345$ & $-0,975$ & $-0,846$ & 1,697 & $-2,423$ & $-0,540$ \\
\hline $\mathrm{C}$ & 0,931 & 0,792 & $-0,842$ & 2,949 & 0,044 & 0,940 & 0,775 & $-0,992$ & 3,513 & 0,421 \\
\hline \multirow[t]{2}{*}{ Q4 } & 0,277 & 0,608 & $-0,150$ & 0,017 & 2,210 & 0,471 & 0,735 & $-0,261$ & 0,141 & 2,527 \\
\hline & \multicolumn{10}{|c|}{ Efeito de ambiente permanente individual } \\
\hline I & $1.825,96$ & 790,904 & $-143,642$ & 109,318 & 80,170 & $1.795,79$ & 778,214 & $-134,600$ & 118,826 & 96,308 \\
\hline $\mathrm{L}$ & 0,840 & 484,975 & 0,064 & $-20,434$ & 23,552 & 0,843 & 474,259 & 3,813 & $-14,147$ & 39,673 \\
\hline Q2 & $-0,402$ & 0,000 & 69,857 & $-2,632$ & $-8,425$ & $-0,351$ & 0,019 & 81,834 & 16,653 & 12,186 \\
\hline $\mathrm{C}$ & 0,243 & $-0,088$ & $-0,030$ & 111,242 & 68,163 & 0,229 & $-0,053$ & 0,150 & 149,684 & 105,766 \\
\hline \multirow[t]{2}{*}{ Q4 } & 0,189 & 0,107 & $-0,101$ & 0,649 & 99,028 & 0,188 & 0,151 & 0,111 & 0,715 & 146,043 \\
\hline & \multicolumn{10}{|c|}{ Efeito de ambiente permanente materno } \\
\hline I & 116,527 & 9,000 & $-25,924$ & 3,422 & $-15,339$ & 125,273 & 9,637 & $-28,401$ & 2,410 & $-17,351$ \\
\hline $\mathrm{L}$ & 0,990 & 0,710 & $-2,002$ & 0,267 & $-1,191$ & 0,588 & 2,144 & $-2,521$ & 0,789 & $-1,868$ \\
\hline Q2 & $-1,000$ & $-0,990$ & 5,767 & $-0,761$ & 3,413 & $-0,994$ & $-0,674$ & 6,519 & $-0,691$ & 4,062 \\
\hline $\mathrm{C}$ & 0,998 & 0,996 & $-0,998$ & 0,101 & $-0,451$ & 0,389 & 0,974 & $-0,489$ & 0,306 & $-0,563$ \\
\hline Q4 & $-0,999$ & $-0,994$ & 0,999 & $-1,000$ & 2,022 & $-0,960$ & $-0,790$ & 0,985 & $-0,631$ & 2,606 \\
\hline
\end{tabular}

\footnotetext{
${ }^{1}$ I = intercepto; L = coeficientes linear; Q2 = coeficiente quadrático; C = coeficiente cúbico; e Q4 = coeficiente quártico.
} 
a) Genética aditiva direta

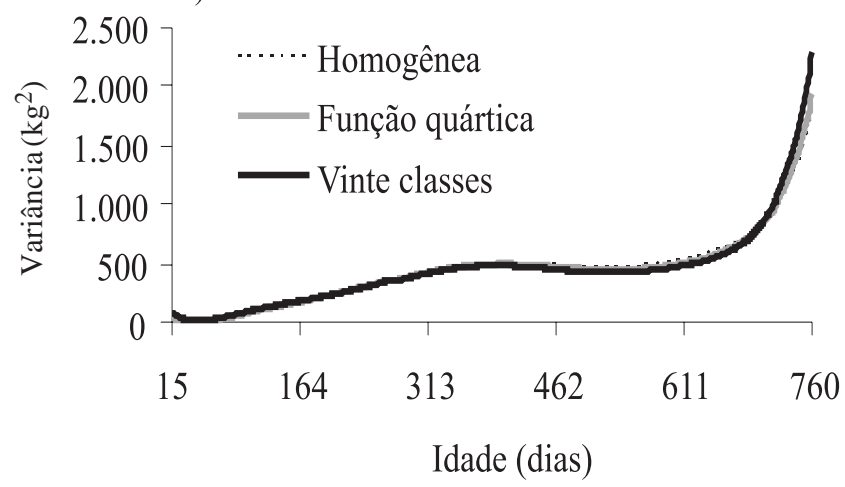

b) Genética aditiva materna

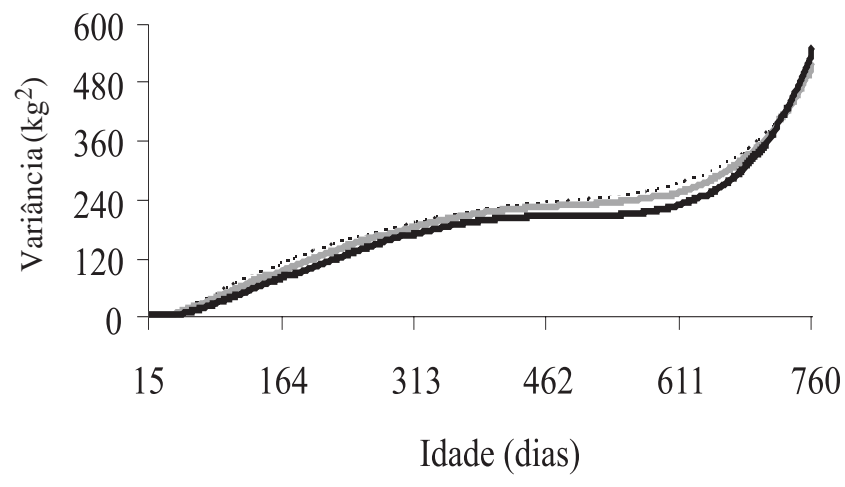

c) Ambiente permanente individual

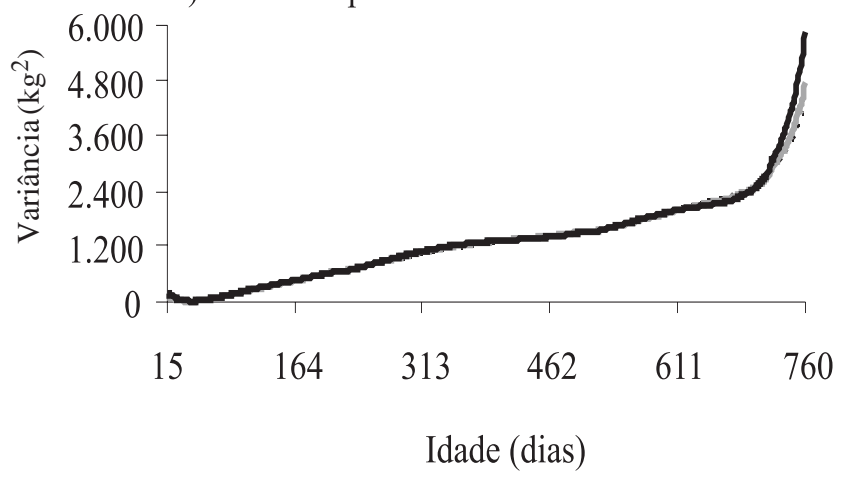

d) Ambiente permanente materno

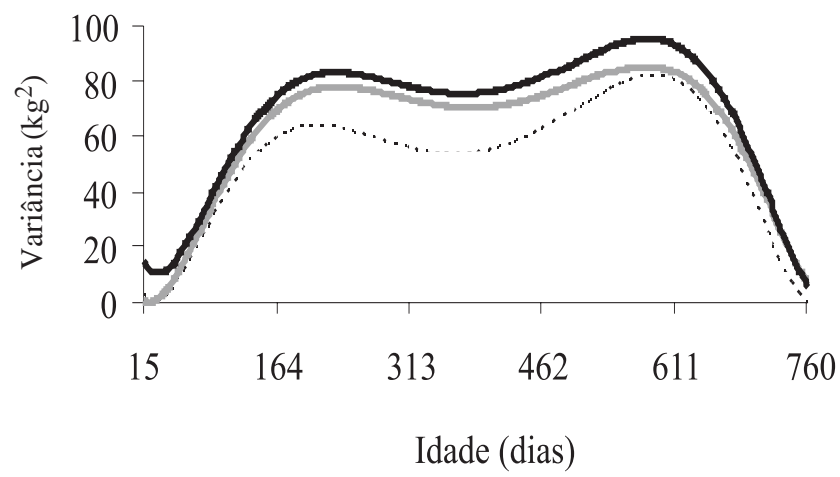

Figura 3 - Variâncias genéticas aditivas diretas e maternas e de ambiente permanente individual e materno para os pesos de bovinos da raça Canchim, de acordo com a estrutura de variância residual.
Foram observados aumentos significativos nas estimativas de variâncias (exceto para a de ambiente permanente materno) para os pesos a partir dos 700 dias de idade (Figura3). Esse comportamento tem sido reportado por outros autores (Meyer, 1999; Albuquerque \& Meyer, 2001; Nobre et al., 2003). É possível que essas alterações súbitas das variâncias tenham sido causadas por falhas no ajuste da trajetória média de crescimento para as idades mais avançadas. Dessa forma, polinômios de Legendre com ordens superiores foram avaliados para modelar a trajetória média de crescimento, mas isso não foi suficiente para melhorar o ajuste nessas idades (dados não apresentados). Outros tipos de polinômios, ou funções segmentadas, poderiam ser avaliados como alternativas para melhorar o ajuste da trajetória de crescimento nas últimas idades disponíveis.

A variância genética aditiva materna foi crescente ao longo do período avaliado (Figura 3). A variância genética aditiva materna e a variância do efeito de ambiente permanente materno sofreram alterações com a utilização de variâncias residuais diferentes ao longo de grande parte do período estudado, principalmente entre 110 e 660 dias (Figura 3). A variância genética aditiva materna aumentou com a utilização da variância residual constante e diminuiu com a utilização de 20 classes. Para a variância do efeito de ambiente permanente materno, ocorreu o contrário, ou seja, as estimativas obtidas considerando a variância residual constante foram menores. As estimativas obtidas com a utilização da função de variância residual quártica foram intermediárias, tanto para a variância genética aditiva materna como para a variância do efeito de ambiente permanente materno. Essas alterações nas variâncias dos efeitos maternos podem ter ocorrido em virtude da dificuldade de separar os efeitos genéticos daqueles não-genéticos.

Não foram avaliados modelos com diferentes ordens para os polinômios e acredita-se que as ordens utilizadas são suficientes para representar as variações, uma vez que poucos estudos consideraram ordens de ajuste superiores a cinco para efeitos fixos e aleatórios (Albuquerque \& Meyer, 2001; Cyrillo, 2003; Santoro etal., 2005; Dias etal., 2006). Essas comparações poderão ser realizadas futuramente para obtenção de modelos menos parametrizados sem prejuízos de ajuste.

Na maior parte do período estudado, as herdabilidades e proporções estimadas nos três modelos para as variâncias residuais foram semelhantes. As maiores diferenças entre as estimativas obtidas com diferentes estruturas de variâncias residuais foram observadas nas idades iniciais, enquanto, após os 240 dias, as diferenças causadas pelas diferentes estruturas de variância residual tenderam a diminuir (Figura 4). 
a) Genética aditiva direta

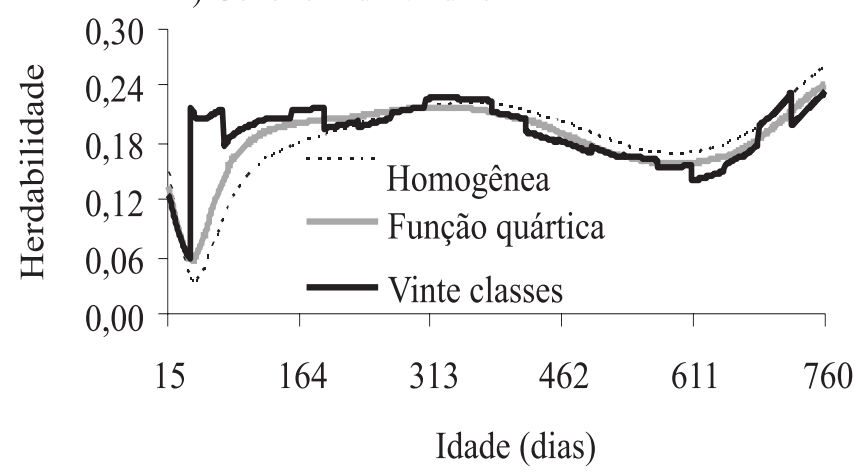

b) Genética aditiva materna

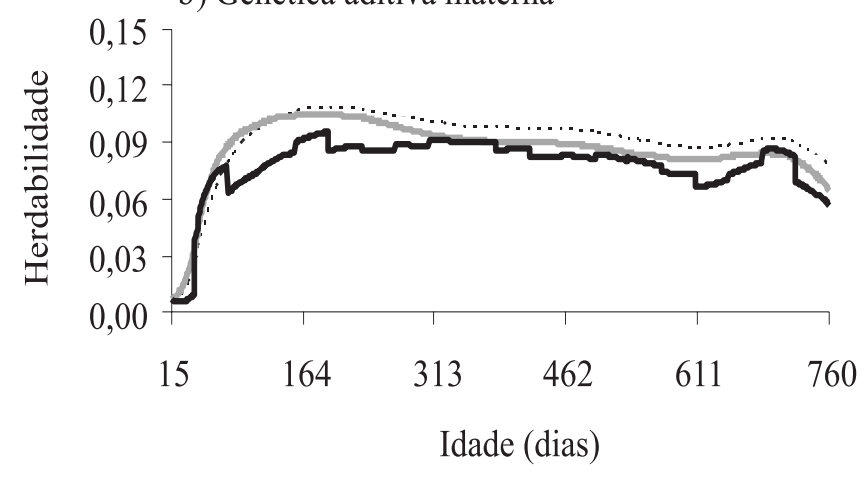

c) Ambiente permanente individual

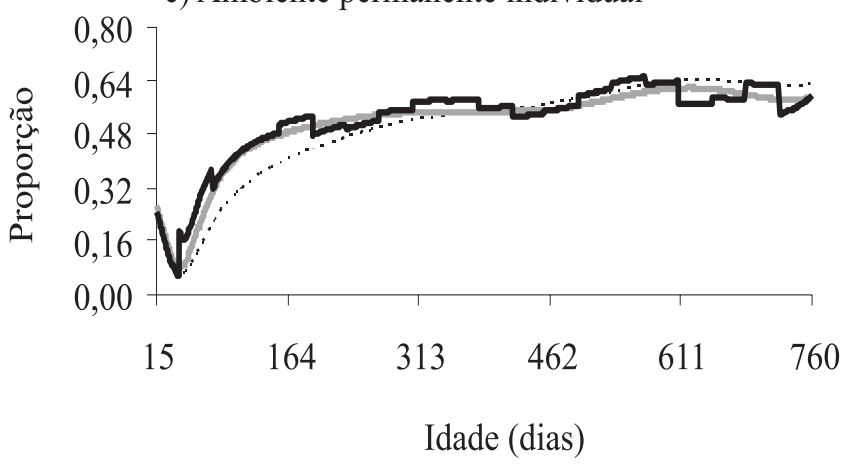

d) Ambiente permanente materno

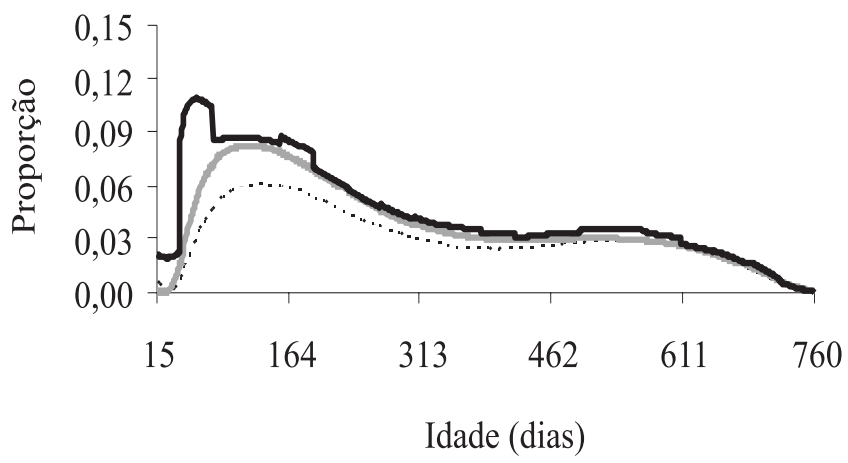

Figura 4 - Herdabilidades direta e materna e proporções da variância fenotípica atribuídas aos efeitos de ambiente permanente individual e materno para os pesos de bovinos da raça Canchim, de acordo com a estrutura de variância residual.
As estimativas de herdabilidade direta se situaram entre 0,16 e 0,25 na maioria das idades consideradas e as maiores estimativas foram obtidas próximo aos 360 dias de idade e ao final do período estudado, confirmando que o critério de seleção utilizado neste rebanho (peso aos 12 meses ajustado para idade da mãe e mês de nascimento) corresponde a uma fase em que a resposta à seleção pode ser maior. As estimativas obtidas para as idades iniciais foram bastante distintas daquelas obtidas para as outras idades, concordando com os resultados encontrados por Cyrillo (2003). Problemas nas estimativas obtidas nas extremidades do período estudado podem ser causados pela utilização de polinômios com ordens de ajuste insuficientes ou inadequados (Meyer, 1999).

As estimativas de herdabilidades diretas foram de 0,20; 0,22; 0,17 e 0,24 para o modelo com variância residual constante; 0,$21 ; 0,22 ; 0,16$ e 0,22 para o modelo com função de variância residual quártica e 0,20; 0,23; 0,16 e 0,21 para o modelo com 20 classes de variâncias residuais para os pesos aos 240, 365, 550 e 730 dias de idade, respectivamente. Mascioli et al. (1996), utilizando o método dos quadrados mínimos, estimaram herdabilidades diretas de 0,47; 0,53; 0,54 e 0,27 para os pesos à desmama, aos 365, 550 e 730 dias de idade em bovinos da raça Canchim, enquanto Mello et al. (2002), utilizando o método da máxima verossimilhança restrita e os dados do mesmo rebanho utilizado neste estudo, estimaram herdabilidades diretas de 0,48 e 0,63 para os pesos aos 240 e 365 dias de idade, respectivamente. Parte dessas diferenças pode ser atribuída ao fato de que, nos modelos de regressão aleatória, foram incluídos o efeito de ambiente permanente individual, que, por sua vez, pode absorver parte da variância genética aditiva direta, levando a reduções nas estimativas de herdabilidade.

As estimativas das proporções da variância fenotípica atribuídas ao efeito de ambiente permanente individual aumentaram a partir de 90 dias de idade e variaram de 0,30 e 0,65. Essa tendência e esses valores são semelhantes aos estimados por Dias et al. (2006) para dados de desempenho de bovinos Tabapuã jovens.

Considerando as estimativas de herdabilidades maternas obtidas com os modelos de variância residual homogênea e 20 classes de variância residual, as maiores estimativas $(0,10$ - 0,12) foram observadas para os pesos próximos aos 160 dias de idade, com tendência de redução após esta idade. Comportamento semelhante foi observado para a proporção da variância fenotípica atribuída ao efeito de ambiente materno permanente. Esses resultados corroboram aqueles obtidos por Dias et al. (2006) e sugerem que a influência da vaca (genótipo e ambiente) sobre o desempenho de suas crias atinge um pico antes da desmama e a partir deste ponto, 
quando o consumo de alimentos sólidos pelo animal supera o consumo de leite, tende a diminuir.

Como esperado, as correlações genéticas foram maiores quando o intervalo de idades foi menor, e superiores a 0,50 na maioria dos pares de idades (Figura 5).

As estimativas das correlações genéticas aditivas diretas entre o peso aos 240 dias e os pesos aos 365, 550 e 730 dias foram, respectivamente, de 0,93; 0,75 e 0,56 (variância residual homogênea); 0,93; 0,73 e 0,56 (função de variância residual quártica); e 0,92; 0,70 e 0,53 (20 classes de variância

Homogênea

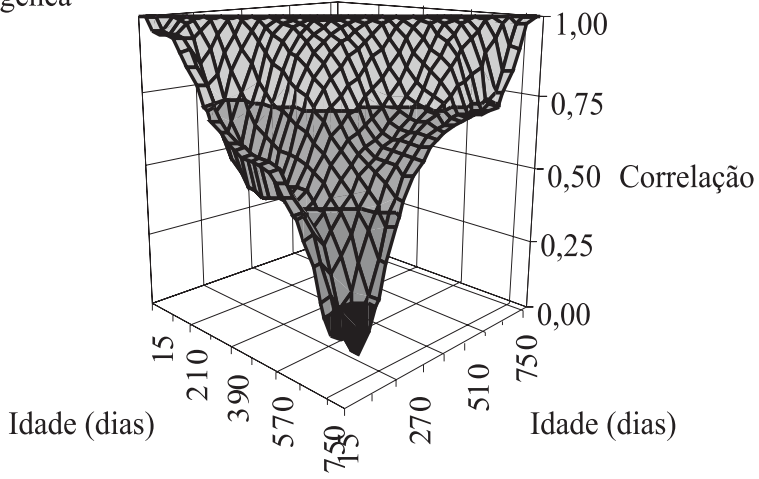

Função quártica

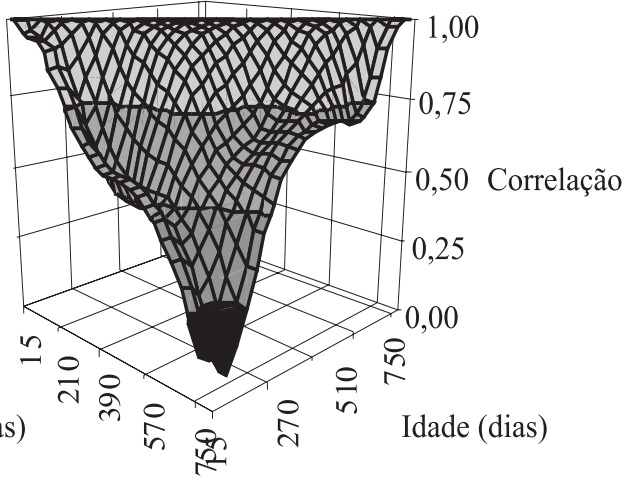

20 classes

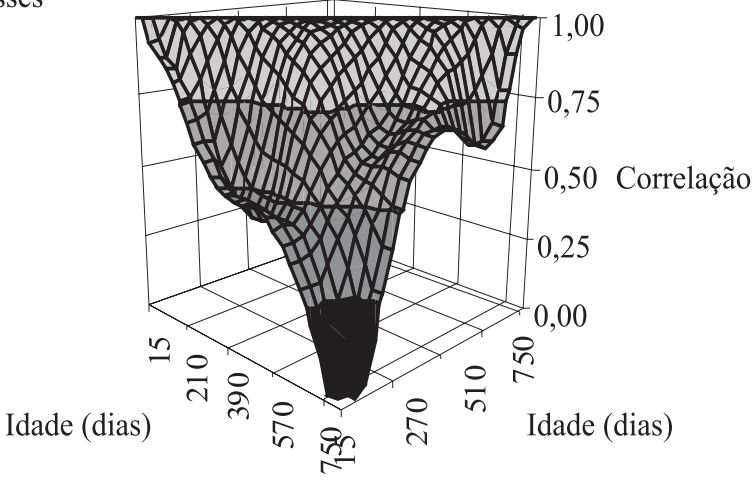

Figura 5 - Correlações genéticas diretas entre os pesos de bovinos da raça Canchim, de acordo com a estrutura de variância residual. residual). Esses valores foram próximos, exceto para as correlações entre os pesos nas idades de 240 e 730 dias, aos estimados por Mascioli et al. (1996), de 0,92; 0,77 e 0,75, respectivamente, o que sugere a possibilidade de respostas correlacionadas para pesos em várias idades quando o critério de seleção é o peso à desmama.

Assumir homogeneidade de variância residual significa admitir que o ambiente temporário afeta igualmente os pesos em todas as idades, o que nem sempre ocorre (Dias et al., 2006). Além disso, diferentes modelagens da variância residual podem proporcionar diferenças nas herdabilidades e razões de variâncias, com possíveis alterações nas ponderações atribuídas ao desempenho próprio, ou de parentes, na avaliação genética.

\section{Conclusões}

As funções de covariância com diferentes estruturas de variância residual podem ser aplicadas para estimação dos componentes de covariância para peso ao longo da vida, porém as estimativas dos parâmetros dependem da estrutura utilizada. O modelo que melhor se ajusta ao peso em diferentes idades de bovinos da raça Canchim é aquele que considera 20 classes heterogêneas. Entretanto, como existem classes com variâncias semelhantes, é possível agrupar classes com variâncias residuais semelhantes e reduzir o número de parâmetros estimados, diminuindo a demanda computacional para o ajuste dos modelos.

\section{Literatura Citada}

ALBUQUERQUE, L.G.; MEYER, K. Estimates of covariance functions for growth from birth to 630 days of age in Nelore cattle. Journal of Animal Science, v.79, n.11, p.2776-2789, 2001.

BOZDOGAN, H. Model selection and Akaike's information criterion (AIC): the general theory and its analytical extensions. Psychometrika, v.52, n.3, p.345-370, 1987.

CYRILLO, J.N.S.G. Estimativas de funções de covariância para crescimento de machos Nelore utilizando modelos de regressão aleatória. 2003. 72f. Tese (Doutorado em Produção Animal) - Faculdade de Ciências Agrárias e Veterinárias/ Universidade Estadual Paulista, Jaboticabal, 2003.

DIAS, L.T.; ALBUQUERQUE, L.G.; TONHATI, H. et al. Estimação de parâmetros genéticos para peso do nascimento aos 550 dias de idade para animais da raça Tabapuã utilizando-se modelos de regressão aleatória. Revista Brasileira de Zootecnia, v.35, n.5, p.1915-1925, 2006.

EL FARO, L.; ALBUQUERQUE, L.G. Utilização de modelos de regressão aleatória para produção de leite no dia do controle, com diferentes estruturas de variâncias residuais. Revista Brasileira de Zootecnia, v.32, n.5, p.1104-1113, 2003.

FRIES, L.A.; ROSO, V.M. Conectabilidade em avaliações genéticas de gado de corte: uma proposta heurística. In: REUNIÃO ANUAL DA SOCIEDADE BRASILEIRA DE ZOOTECNIA, 34., 1997, Juiz de Fora. Anais... Juiz de Fora: Sociedade Brasileira de Zootecnia, 1997. p.159-161. 
MASCIOLI, A.S.; ALENCAR, M.M.; BARBOSA, P.F. et al. Estimativas de parâmetros genéticos e proposição de critérios de seleção para pesos na raça Canchim. Revista da Sociedade Brasileira de Zootecnia, v.25, n.1, p.72-82, 1996.

MELLO, S.P.; ALENCAR M.M.; SILVA, L.O.C. et al. Estimativas de (co)variâncias e tendências genéticas para pesos em um rebanho Canchim. Revista Brasileira de Zootecnia, v.31, n.4, p.1707-1714, 2002.

MEYER, K. DFREML version 3.0b user note. Armidale: Animal Genetics and Breeding Unit, 1998. 33p.

MEYER, K. Estimates of genetic and phenotypic covariance functions for postweaning growth and mature weight of beef cows. Journal of Animal Breeding and Genetics, v.116, n.3, p.181-205, 1999.

MEYER, K. Random regression to model phenotypic variation in monthly weights of Australian beef cows. Livestock Production Science, v.65, n.1, p.19-38, 2000.

MEYER, K. Scope for random regression model in genetic evaluation of beef cattle for growth. Livestock Production Science, v.86, n.1-3, p.69-83, 2004.
NOBRE, P.R.C.; MISZTAL, I.; TSURUTA, S. et al. Analyses of growth curves of Nellore cattle by multiple-trait and random regression models. Journal of Animal Science, v.81, n.4 p.918-926, 2003.

SANTORO, K.R.; BARBOSA, S.B.P.; SANTOS, E.S. et al. Uso de funções de covariância na descrição do crescimento de bovinos Nelore criados no Estado de Pernambuco. Revista Brasileira de Zootecnia, v.34, n.6, p.2290-2297, 2005 (supl.).

SAKAGUTI, E.S.; SILVA, M.A.; QUAAS, R.L. et al. Avaliação do crescimento de bovinos jovens da raça Tabapuã, por meio de análises de funções de covariâncias. Revista Brasileira de Zootecnia, v.32, n.4, p.864-874, 2003.

SORENSEN, D.; GIANOLA, D. Likelihood, bayesian, and MCMC methods in quantitative genetics. New York: Sringer-Verlag New York Inc, 2002. 740p.

WOLFINGER, R.D. Covariance structure selection in general mixed models. Communications in Statistics - Simulation, v.22, n.4, p.1079-1106, 1993 